\title{
Cochrane Review Summary: psychosocial and psychological interventions for preventing postpartum depression
}

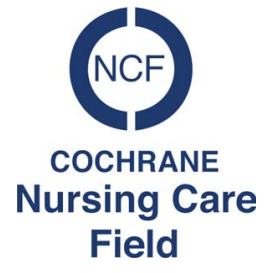

\section{Daksha Trivedi}

Senior Research Fellow, Evidence Based Practice, Centre for Research in Primary and Community Care, University of Hertfordshire, Hatfield, UK

Key words: interventions; postpartum depression; psychological; psychosocial

First published online 13 May 2014

\section{Review question}

1. To assess the effectiveness of specific psychosocial and psychological interventions in reducing the risk of postpartum depression in mothers and their families.

2. To examine the influence of variations in intervention types, providers, modes, duration, onset and high risk women

\section{Relevance to primary care and nursing}

Primary care teams, including nurses, midwives, health visitors and general practitioners are involved in identifying and managing depression during antenatal and postpartum care. The National Institute for Health and Care Excellence (NICE) has issued a guidance report for health care professionals on routine postnatal care, which includes depression (NICE, 2006). It is important to identify effective intervention for postpartum care.

\section{Characteristics of the evidence}

This Cochrane review contained 28 randomised controlled trials, which included 16912 pregnant women and new mothers (less than six weeks postpartum) at no known risk or at risk of developing postpartum depression (Dennis and

Correspondence to: Dr Daksha Trivedi, Senior Research Fellow, Evidence Based Practice, Centre for Research in Primary and Community Care, University of Hertfordshire, College Lane, Hatfield, AL10 9AB, UK. Email: d.trivedi@herts.ac.uk
Dowswell, 2013). They were conducted in Australia (12), United Kingdom (seven), United States (four), China (two), and Canada, Germany and India (three), respectively. Interventions needed to be non-pharmacological, aiming to reduce the risk of developing postpartum depression, compared with any form of standard or usual care. They included psycho-educational strategies, cognitive behavioural therapy (CBT), interpersonal psychotherapy, non-directive counselling, psychological debriefing and various supportive interactions. They were delivered by professionals (eg nurse, physician, psychiatrist, psychologist) or trained volunteers via telephone sessions, home or clinic visits, or individual or group sessions given antenatally and/or up to four weeks postpartum. The type, intensity and duration of interventions varied considerably.

\section{Summary of key evidence}

Most trials were of good quality (low risk of bias). Outcomes were defined in various ways and were analysed at final assessment and at four time points across the postpartum period: immediate (zero to eight weeks); short term ( 9 to 16 weeks); intermediate (17 to 24 weeks); long term (more than 24 weeks).

Primary outcomes were depressive symptomology, clinical diagnosis of depression (dichotomous) and mean depression scores. Secondary outcomes included various maternal, infant and family outcomes. Data were combined in a meta-analysis. 


\section{Psychosocial and psychological interventions}

There was a significant beneficial effect $(22 \%)$ on depressive symptomatology (20 trials, $n=14$ 727) that was sustained in the immediate, shortterm and long-term period. There was a significant overall preventative effect $(50 \%$; five trials, $n=939)$ and a short-term effect on clinical diagnosis of depression, with a small effect on mean scores. There was no evidence of benefit on mortality, maternal-infant measure, Parental Stress Index, maternal perceptions of support or social support, although mean anxiety scores reduced significantly (four trials, $n=815$ ). Stress levels improved (one trial, $n=103$ ) but not in the long term. Satisfaction with care improved significantly (four trials, $n=3014$ ), although the mean effect was not significant. There was no evidence of longterm effect on infants not fully immunised, infant development or child abuse, although the latter reported a beneficial effect in the immediate postpartum period (one trial, $n=176$ ). There was no significant effect on marital discord scores.

\section{Psychosocial interventions}

There was an overall beneficial effect (17\%) on depressive symptomatology (12 trials, $n=11322$ ), which remained from short term to longer term ( $40 \%$; three trials, $n=1385$ ), a significant effect on clinical depression ( $48 \%$; three trials, $n=867)$ and no significant effect on mean scores.

\section{Psychological interventions}

There was a significant reduction $(39 \%)$ in depressive symptomatology (eight trials, $n=3405$ ) and a short-term effect, with no significant effect on clinical depression or means.

\section{Sub-group analysis}

\section{Variations in interventions}

For psychosocial interventions, professionalbased home visits showed benefit (44\%; two trials, $n=1262$ ) as did postpartum lay-based telephone support ( $46 \%$; one trial, $n=612$ ). Antenatal and postnatal classes, postpartum laybased home visits, early postpartum follow-up or continuity/model of care showed no significant effect. Interpersonal psychotherapy reported a protective effect on mean scores (five trials, $n=366)$ but psychological debriefing and CBT showed no overall effect. Professionally based provider interventions showed a reduction $(22 \%)$ in depressive symptomatology ( 15 trials, $n=6790$ ) with no significant effect on clinical depression or means. Lay-based interventions showed a significant protective effect $(30 \%)$ for depressive symptomatology (four trials, $n=1723$ ), a shortterm effect (48\%) in clinical depression (two trials, $n=677)$ and no significant mean effect.

\section{Professionally based provider}

There was no significant effect on depressive symptomatology or mean scores for interventions provided by nurses, physicians, midwives or mental health professional.

\section{Intervention mode}

Individually based interventions showed a significant reduction $(25 \%)$ in depressive symptomatology (14 trials, $n=12914)$, a significant reduction (47\%) in clinical depression (three trials, $n=714$ ) and no significant mean effect. Groupbased interventions showed no significant effect on depressive symptomatology, clinical depression or mean scores.

\section{Intervention duration}

Single-contact interventions showed no significant effect on depressive symptomatology or mean scores. Multiple-contacts showed a significant reduction $(22 \%)$ in depressive symptomatology (16 trials, $n=11850$ ), clinical depression ( $52 \%$; five trials, $n=939$ ) and a small mean effect.

\section{Intervention onset}

Antenatal-only intervention showed no mean effect; antenatal followed by postnatal interventions showed no significant effect on depressive symptomatology or means scores, and a significant reduction $(56 \%)$ in clinical depression (three trials, $n=292$ ), although two trials were of poor methodological quality. Postnatal-only interventions showed a significant protective effect $(27 \%)$ on depressive symptomatology ( 12 trials, $n=12786$ ), with no significant effects on other outcomes.

\section{Interventions for at-risk women}

There was a significant reduction $(34 \%)$ in depressive symptomatology (eight trials, $n=1853$ ), mean scores (seven trials, $n=1087$ ) and clinical 
depression (52\%; five trials, $n=939)$, although removing three poor quality trials showed a non-significant effect. Women from the general population showed no significant effect.

\section{Implications for practice}

There is good evidence to consider recommendations for intensive, professionally based postpartum home visits, telephone-based peer support and interpersonal psychotherapy. There is not enough evidence to recommend antenatal and postnatal classes, early postpartum follow-up care, lay-based interventions at home, CBT, psychological debriefing or continuity of care models. Targeting at risk mothers may be more beneficial than all women.

\section{Implications for research}

Research needs to examine the components of psychosocial interventions that reduce postpartum depression and the use of a screening tool for early detection and flexible, individualised postnatal care. Further evaluations include a peer-led telephone-based support among new mothers with early depression, individually tailored and lay-based interventions, delivery modes and providers of interpersonal psychotherapy, targeting vulnerable groups and partners of mothers and economic evaluations.

\section{Acknowledgements}

The author is a member of the Cochrane Nursing Care Field (CNCF).

\section{Financial Support}

This research received no specific grant from any funding agency, commercial or not-for-profit sectors.

\section{Coflicts of Interest}

None.

\section{Ethical Standards}

Not applicable. This is a summary based on secondary research and is not dealing with animals

\section{References}

National Institute for Health and Clinical Excellence (NICE). 2006: Clinical guideline 37. Routine postnatal care of women and their babies. London: NICE. Retrieved from http:// www.nice.org.uk/nicemedia/live/10988/30144/30144.pdf

Dennis, C.L., Dowswell, T. 2013: Psychosocial and psychological interventions for preventing postpartum depression. Cochrane Database of Systematic Reviews 4, CD001134 